\title{
Physiological Identification of Afferent Fibers and Postsynaptic Sensory Neurons in the Spinal Cord of the Intact, Awake Cat
}

\author{
L. S. SORKIN, ${ }^{*, 1}$ T. J. MORROW, ${ }^{*}$ AND K. L. CASEY ${ }^{\dagger}, 2$ \\ Departments of Physiology* and Neurology, $\dagger$ University of Michigan and Neurology Research \\ Laboratories (Neurophysiology), Veterans Administration Medical Center, \\ Ann Arbor, Michigan 48105
}

Received March 24, 1987; revision received July 20, 1987

\begin{abstract}
A method was developed to record from spinal cord cells in the awake, intact, partially restrained cat. Units were classified as afferent fibers or postsynaptic cells based on their ability to follow $100-\mathrm{Hz}$ peripheral stimulation, the duration and configuration of the action potential waveform, and the number of spikes evoked by a single electrical pulse. These criteria are supported by independent observations of the location of the recording site, size of the receptive field, and adequate stimulus. Of 84 cutaneously activated units, 29 were classified as afferent fibers, 28 as postsynaptic cells, and 27 were not classified. No cutaneously activated unit was spontaneously active. In contrast, all 28 units (7 postsynaptic and 21 not classified) responding to joint position or movement were spontaneously active (5 to $40 \mathrm{~Hz}$ ). No unit responded to both cutaneous and proprioceptive inputs. Evidence for convergence of cutaneous input from different types of receptors was limited to five postsynaptic neurons that responded to hair movement and to stimuli applied to the skin; two of these cells responded differentially to noxious pinch. Two of 24 postsynaptic cutaneous units ceased responding to electrical cutaneous stimuli when the cat was eating. The responses of 29 primary afferent fibers were not altered by the behavior of the cat. These results suggest that, in the awake cat (i) criteria based on neuronal responsiveness and action potential waveform can be used to distinguish adequately between afferent fibers and postsynaptic cells; and (ii) there is a tonic inhibitory control, greater than in the anesthetized or spinally transected cat, that varies with behavioral state and is directed primarily at spinal neurons receiving cutaneous input. 1988 Academic Press, Inc.
\end{abstract}

\footnotetext{
${ }^{1}$ Address correspondence to: Dr. Linda S. Sorkin, Marine Biomedical Institute, University of Texas Medical Branch, Galveston, TX 77550.

${ }^{2}$ Supported by the Veterans Administration and National Institutes of Health grants NS 12015 and 5T32-GM07106.
} 


\section{INTRODUCTION}

Knowledge of the responses of sensory cells in the spinal cord has been obtained mainly from anesthetized or surgically reduced animals. However, the type of preparation used, whether anesthetized, spinal, or decerebrate, is known to affect the baseline levels of activity $(24,36)$, receptive field sizes (1), and adequate stimuli $(3,5,36)$ of sensory cells. The limited data available from intact, awake animals indicate that most spinal cord somatosensory cells in the awake rat (37), cat $(11,12)$, and monkey (2) exhibit little or no spontaneous activity. Furthermore, single-unit recordings in the dorsal horn of the rat (36) and in the caudal part of the spinal trigeminal nucleus of the monkey (14) indicate that both the behavioral relevance of the stimulus and attention can affect neuronal excitability, threshold, and receptive field size.

A complete understanding of the normal sensory neurophysiology of the mammalian spinal cord will require additional recordings from intact, awake animals. An adequate interpretation of such studies also requires establishing and testing neurophysiologic criteria to distinguish between primary afferent fibers and postsynaptic cells. This requirement becomes nontrivial in the awake animal because it is not possible to use all the tests that are applied in anesthetized or surgically reduced preparations. In the experiments reported hcre, we systematically investigated the physiologic properties of units that were classified as afferent fibers or postsynaptic cells and compared their response characteristics with those reported in acute experiments. We also examined the effects of changes in behavioral state on the excitability of spinal sensory neurons.

Some results of these experiments have been reported elsewhere $(31,32)$.

\section{METHODS}

All aspects of animal care and housing and all experimental procedures were conducted in accord with standards and guidelines established by the American Association for the Accreditation of Laboratory Animal Care.

Twelve female cats were maintained on tap water and dry food at approximately $85 \%$ of ad libitum weight. Each cat was trained to lie quietly in a flexible body cast and to accept small amounts of liquified cat food delivered periodically to a food cup throughout each recording session. Details of the apparatus are reported elsewhere (9). During the restraint period, cats were conditioned to accept moderate amounts of hindquarter movement and palpation as well as the occasional subcutaneous insertion of 25 -gauge needle electrodes, which were used to deliver innocuous electrical stimulation.

Cats received penicillin (150,000 units, i.m.) for 10 days starting the day before surgery. $\Lambda$ fter preanesthetic doses of ketaminc hydrochloride $(10 \mathrm{mg} /$ $\mathrm{kg}, \mathrm{i} . \mathrm{m}$.) and atropine sulfate $(0.2 \mathrm{mg}$, s.c. $)$, anesthesia was achieved with sufficient sodium pentobarbital to eliminate flexor reflexes. During surgery, 
supplemental barbiturate and fluids were administered via a catheter in the cephalic vein. The total anesthetic dose rarely exceeded $35 \mathrm{mg} / \mathrm{kg}$ i.v. Rectal temperature was monitored and maintained at $38^{\circ} \mathrm{C}$.

Lumbar vertebral segments 3 through 5 were exposed and cleared of all soft tissue down to and including the lateral and accessory processes. A ground screw was positioned in the bone at $L_{3}$ and a reference electrode was inserted into the underlying spinal cord. Both leads were fixed in place with cold-cure dental cement and crimped to gold male microplug contacts which were inserted into a female connector. A partial laminectomy was made at the $\mathrm{L}_{4}$ vertebra to create an opening slightly larger than the mounting sleeve of the chronic microelectrode device [modified from Morrow (26)].

The mounting sleeve was centered over the dorsal vein of the $L_{5-6}$ spinal segments. Some of the earlier implants were placed in more caudal positions. A saline-soaked Gelfoam sponge was placed on the dura and the mounting sleeve was lowered until it was touching the sponge. Silicone rubber was applied around the edges of the implant to seal the opening and to protect any exposed dura. A thin coat of dental cement was used to secure the plastic sleeve to the bone. Plastic sheeting material was used to protect the exposed muscle. Dental acrylic was layered around the mounting sleeve and connector and over the vertebrae; all three sets of accessory processes were included to ensure immobilization of the lower lumbar spine and to reduce cord movement. After the acrylic had cured, the plastic sheeting was removed and the wound was thoroughly cleaned and sutured. For the duration of the experiment, the incision was cleaned daily with a $4 \%$ Combiotic solution. Topical lidocaine was applied in the first few postsurgical days. Within 5 postoperative days, cats walked about the laboratory in no apparent distress, spontaneously ate in sufficient amounts to maintain weight, groomed themselves, and accepted petting.

Data collection commenced after a recovery period of 1 to 2 weeks. Standard electrophysiologic equipment was used for extracellular recording. Stainless-steel microelectrodes with an in vivo impedance of 3 to $10 \mathrm{M} \Omega$ (tested at $300 \mathrm{~Hz}$ ) were placed in a drive screw inserted into the microelectrode device and passed through a Silastic rubber sheet at the bottom of the mounting sleeve. The drive screw could be positioned so that the electrode track began on an arc 1 or $2 \mathrm{~mm}$ from the center of the mounting sleeve. At the beginning of each recording session, the cat was placed in the cast. The headstage was plugged into a connector on the cat's back.

Because spontaneously active units were rarely seen, manual stimuli (touch, hair movement, etc.) were applied continually to activate and locate cells. Searching for receptive fields was in a caudal to rostral direction and did not include areas in contact with the restraint device. This procedure eliminated the possibility of examining cells with habituated responses. The search procedure was carried out only when the cat was not eating. Moderate 

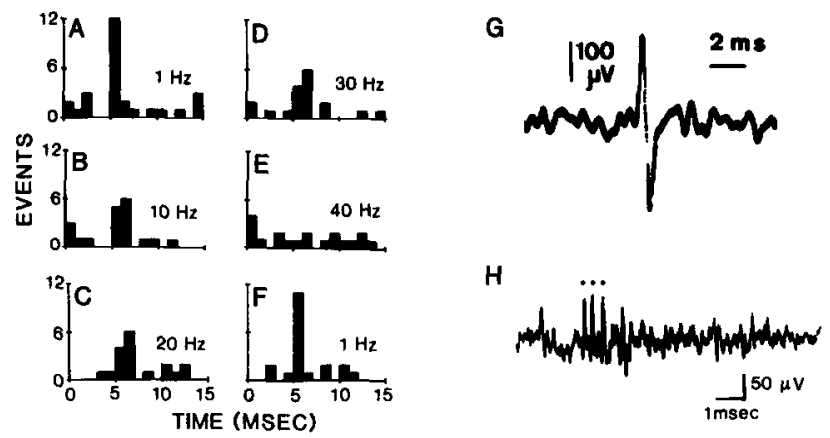

FIG. 1. Response characteristics of units classified as postsynaptic. A through F-poststimulus histograms of the responses of a single unit to electrical stimulation of receptive field at indicated frequencies. Bin width equals $1 \mathrm{~ms}$. Note response failure at $40 \mathrm{~Hz}(\mathrm{E})$ and restoration of response when stimulus frequency was returned to $1 \mathrm{~Hz}(\mathrm{~F})$. G-waveform and duration of postsynaptic spike. $\mathrm{H}$-burst response of a third postsynaptic unit to single-shock stimulation of receptive field.

movements of the head, hind limbs, or tail did not interfere with electrophysiologic recording.

When a unit was encountered, its response to several types of natural somatic stimuli including hair movements, touch, tap, and pinch was tested and the receptive field was determined. Pinch was sufficient to cause withdrawal and/or vocalization in an untrained animal. Attempts were made to drive the units electrically using needle electrodes placed in the receptive field. Response latencies and conduction distances were measured. Units were considered postsynaptic if they showed any one of the following: (i) failed to respond with a constant latency to each of the stimuli delivered at frequencies of 1 to $100 \mathrm{~Hz}$; (ii) displayed a biphasic waveform with a duration of $0.5 \mathrm{~ms}$ or longer (18) (Fig. 1); or (iii) responded to a single 0.1 -ms electrical pulse with repetitive firing (Fig. 1). Units that responded with a fixed latency to each stimulus of a $100-\mathrm{Hz}$ train were classified as presynaptic (afferent fibers). It was not possible to classify all units because all tests could not always be completed while each unit was isolated adequately.

The threshold and responses of each cell were examined under two behavioral conditions: while the cat was eating and while it was not eating. Poststimulus histograms of unit responses to each of 25 electrical stimuli were compiled by a microprocessor. A chart recorder displayed an integrated record of unit responses to natural stimuli. A modified goniometer was used to measure velocity of joint movement and absolute joint angle. All data were stored on magnetic tape for further analysis.

At the end of each session, the electrode remained in the spinal cord, a protective cap was secured over the electrode, and the cat was returned to its 
TABLE 1

Classification of Somatosensory Neurons

\begin{tabular}{lcccr}
\hline & \multicolumn{2}{c}{ Afferent postsynaptic } & & \\
\cline { 2 - 5 } & Fibers & Neurons & Unclassified & Total \\
\hline Cutaneous & 29 & 28 & 27 & 84 \\
Joint/movement & & 7 & 21 & 28 \\
Total & 29 & 35 & 48 & 112 \\
\hline
\end{tabular}

cage. This procedure permitted a thorough and prolonged exploration of each track over several recording sessions. Microelectrodes left in the cat for periods of 1 to 2 weeks produced no obvious damage to the spinal cord (see Fig. 7).

Electrolytic lesions were made at the completion of each electrode track. After scveral tracks had been completed, the cats were decply anesthetized and transcardially perfused with physiological saline followed by a solution of $10 \%$ Formalin and potassium ferrocyanide. Frozen sections of spinal cord were mounted and stained with cresyl violet. The electrode tracks were reconstructed from projected images of the histologic sections.

\section{RESULTS}

A total of 112 spinal cord units that could be driven reliably by natural stimuli were isolated and studied (Table 1). Ten additional units were spontaneously active and sufficiently well isolated for study, but no adequate stimulus could be found. These units are not included in this study. We could not rule out the possibility that the receptive fields of these cells were in contact with the restraining device which covered the abdomen and anterior aspect of the thighs (9).

None of the 84 units that responded to natural cutaneous stimuli was spontaneously active. Twenty-nine (34\%) of these units responded at a fixed latency to each of the electrical stimuli delivered to the receptive fields at frequencies of $100 \mathrm{~Hz}$ or more and were accordingly classified as afferent fibers. Twenty-eight units (33\%) were classified as postsynaptic because they failed to follow a high-frequency stimulus ( 20 cells), because of waveform shape and duration, or because they discharged repetitively to a single electrical pulse applied to the receptive field ( 8 cells). One of the units with a biphasic, long-duration waveform was classified as a postsynaptic unit although it also followed $100-\mathrm{Hz}$ stimulation with a constant latency. The remaining 27 $(32 \%)$ cutaneously responsive units could not be classified with the present criteria. 
Adequate Stimulus. All 29 units classified as cutaneous afferent fibers responded to only one type of input; $19(66 \%)$ emitted rapidly adapting responses to hair movement, and $10(34 \%)$ fired only in response to innocuous levels of touch or pressure on the skin. However, $23(82 \%)$ of the 28 cutaneous units that were classified as postsynaptic neurons also responded to only one type of input; 14 responded to hair movement, seven to touch of the skin, and one to movement of a single claw. One high-threshold cell, which responded to pinprick or noxious levels of pinch, was found when needle electrodes were inserted into the skin to excite a different unit. The 27 unclassified units also responded to either hair movement or skin pressure but never to both types of stimulation.

Only five units, all classified as postsynaptic, responded to more than one type of stimulus. Three units responded differentially to touch and hair movement. Hair movement caused these cells to discharge at a low frequency; application of pressure to the receptive field generated a higher peak firing frequency. Further increasing the intensity of stimulation to a noxious (withdrawal-eliciting) level produced no change in the response (Fig. 2A). Two other postsynaptic units exhibited a graded response to stimuli of increasing strength up to and including the noxious range (Fig. 2B). These multireceptive cells, like all the pressure responsive cells observed, responded to pressure with a brief phasic component followed by a lower frequency, sustained tonic phase.

In addition to the cutaneously responsive units, 28 additional neurons ( $25 \%$ of the total) fired in response to joint position or movement and could not be driven by electrical stimulation in the awake animal. None of these proprioceptive units received cutaneous input and all were spontaneously active. Their resting firing frequencies ranged from 5 to $40 \mathrm{~Hz}$. Seven of these units were classified as postsynaptic based on waveform criteria. The remainder were unclassified. Most of the proprioceptive units had a rapidly adapting response to joint movement. Some units responded more vigorously to either flexion or extension; most fired an equivalent burst in response to either stimulus. Three cells showed sustained changes in activity during a maintained joint position (Fig. 2C).

Receptive Fields. Receptive fields were classified as (1) small (less than 2 $\mathrm{cm}^{2}$ ); (2) medium (less than half of the limb); and (3) large (greater than half of the limb or on two or more body parts. Receptive field size varied with location; proximal receptive fields tended to be larger than distal ones; and those on the tail, for the most part, were larger than those on the limb.

Figure 3 shows the distribution of receptive field sizes among primary afferents, postsynaptic cells, and unclassified units. Most primary afferent fibers had small $(N=18)$ or medium $(N=10)$ size ipsilateral receptive fields located on the tail $(N=10)$, below the knee $(N=8)$, or on the trunk and/or thigh $(N=11)$. The only afferent fiber with a large receptive field was driven 
A

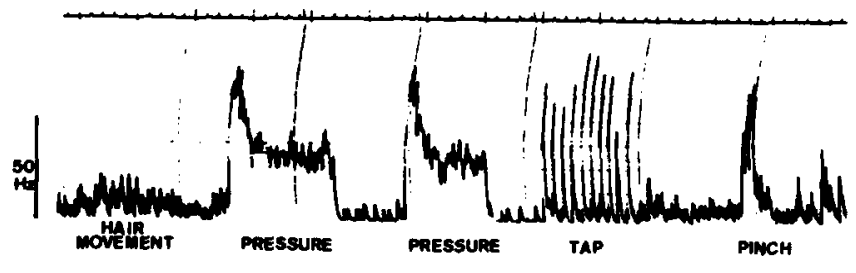

B
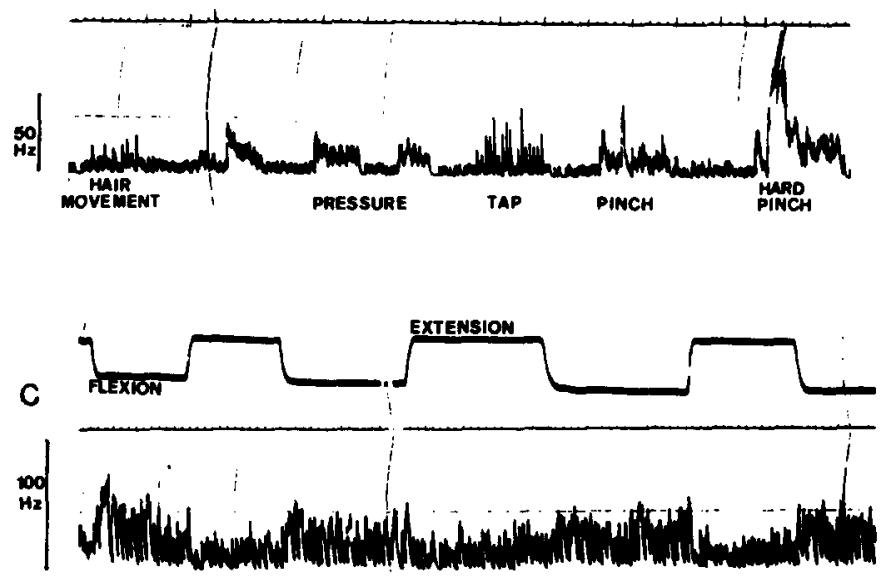

FIG. 2. A and B-Differential responses of postsynaptic units to different forms of natural somatic stimuli. Chart recordings of integrated output of frequency-to-voltage converter. Aunit responded to hair movement but gentle steady pressure on skin elicited higher sustained response; noxious pinch was no more effective than pressure. B-unit with differential responses similar to those shown in A but with additional differential response to noxious pinch. $\mathrm{C}$ unclassified unit with sustained changes in activity (bottom trace) induced by maintained flexion (excitation) and extension (suppression) of leg at the knee joint. Top trace: output of goniometer. Time marks: $1 \mathrm{~s}$.

by hair movement from two discontinuous small fields located on the trunk and thigh. This fiber was driven from both locations at $180 \mathrm{~Hz}$ with no change in latency.

Receptive field sizes of the postsynaptic cells were more evenly distributed, but the majority were medium $(N=10)$ and large $(N=12)$. Receptive fields of postsynaptic cells were usually, but not always, larger than those of primary afferents at the same locus. Frequently, receptive fields of postsynaptic cells that included large portions of the tail extended onto the thigh or rump; this was not true of primary afferent fiber receptive fields.

Most unclassified units had medium size receptive fields $(N=15)$; seven were small and four were large, two of which included portions of both the tail and thigh. 


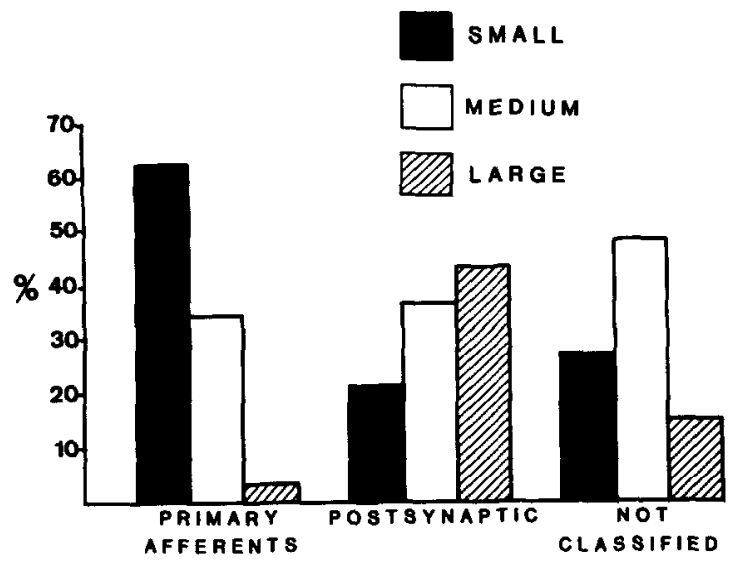

FIG. 3. Distribution of receptivc field sizes among units classified as primary afferents, postsynaptic neurons, or unclassified. Small: less than $2 \mathrm{~cm}^{2}$. Medium: less than one-half of a limb. Large: more than one-half of a limb or located on two or more body parts.

Afferent Conduction Velocity. The average conduction velocity for the cutaneous afferent fibers (Fig. 4A) was $53 \mathrm{~m} / \mathrm{s}$. The cells activated by hair movement had faster average conduction velocities $(58 \mathrm{~m} / \mathrm{s})$ than those that responded to skin stimulation $(41 \mathrm{~m} / \mathrm{s})$, although their distributions showed considerable overlap (Fig. 4C, D). The conduction velocity distribution between the two groups was different at the $P=0.025$ level of significance (Mann-Whitney $U$ test).
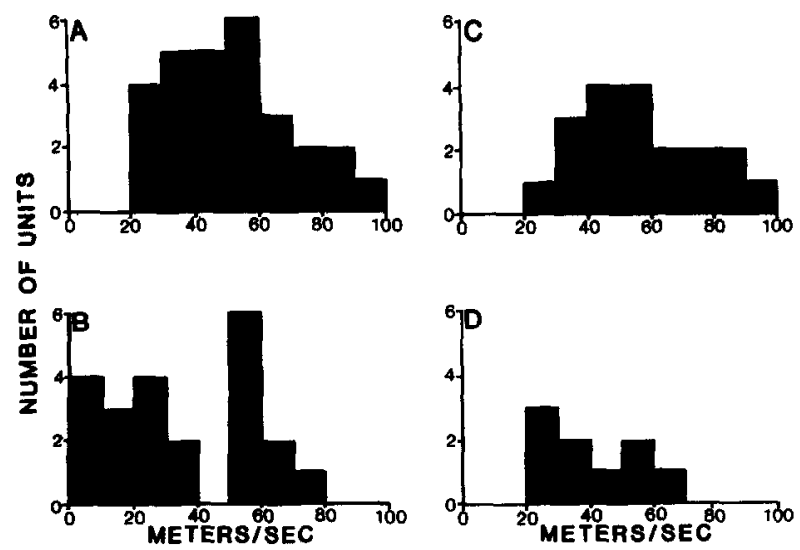

FIG. 4. Distribution of conduction velocities of A-all cutaneous primary afferents; B-all postsynaptic units; $C$ - primary afferents responding only to hair movement; and D-primary afferents responding only to stimulation of the skin. 


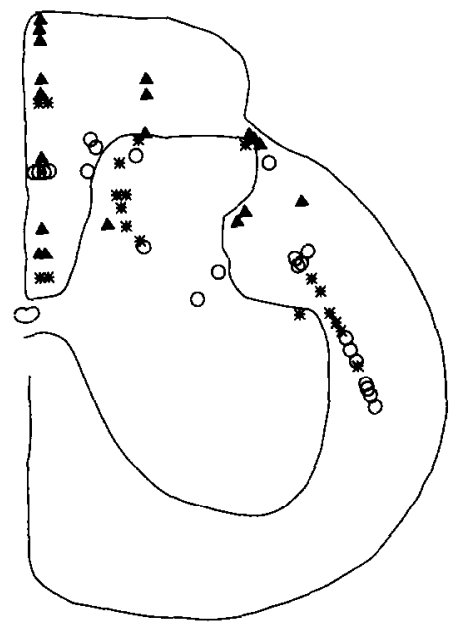

FIG. 5. Recording sites of primary afferent fibers, (solid triangles), postsynaptic cells (asterisks), and unclassified units (open circles) shown on drawings of coronal hemisection of lumbar cord. Reconstruction of loci based on electrolytic lesions made with microelectrode.

The average conduction velocity of postsynaptic units (Fig. 4B) was $37 \mathrm{~m} /$ s. No allowance was made for synaptic delay or central conduction timc. Both as a whole population and within a modality, postsynaptic cells had a slower conduction velocity than did the primary afferent fibers. For the combined population of identified presynaptic fibers and postsynaptic cells, only postsynaptic cells $(N=7)$ had conduction velocities of less than 20 $\mathrm{m} / \mathrm{s}$. Four of the unclassified units had conduction velocities of less than $20 \mathrm{~m} / \mathrm{s}$.

Recording Sites. The locations of 75 recording sites were verified histologically. Fourteen sites were located in sacral and 61 in lumbar segments of the spinal cord. All but one of the afferent fiber recording sites were found in white matter. Postsynaptic units were distributed throughout the dorsal portion of the spinal cord. In the lumbar cord, about half the postsynaptic cells were in gray matter (Fig. 5). All postsynaptic units recorded from the sacral cord were in gray matter.

All four postsynaptic dorsal column fibers had small receptive fields; two were multireceptive and had an increased response to stimuli of increasing intensity. Within the dorsal columns, primary afferent hair fibers were located dorsal to touch/pressure fibers as was described elsewhere (33). Units that responded differentially to stimuli of various intensitics were locatcd either in the dorsal columns or in the deeper laminae of the dorsal horn.

Figure 6 shows the reconstruction of eight recording sites on a single track. Units 1 and 2 were recorded from white matter, followed $100-\mathrm{Hz}$ stimulation, and responded to a single modality. Units 4 through 8 were located in 
A

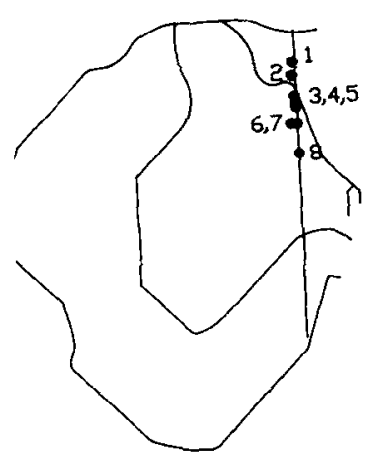

B.

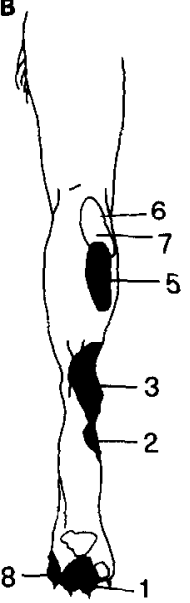

C

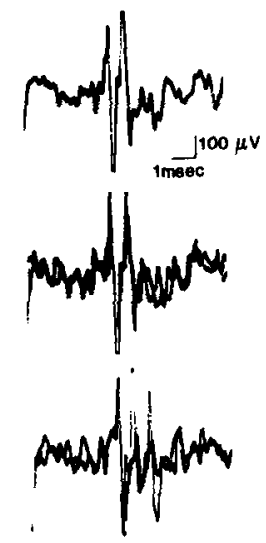

FIG. 6. Distribution of unit response properties and receptive fields along an electrode track. A-loci of recording sites for eight units responding to pressure (units 1,7 , and 8 ), hair movement (units 2, 3, and 6), knee flexion (unit 4), and hair movement, pressure, and pinch (unit 5). B-receptive field sizes and locations for each of the seven cutaneous units. C-postsynaptic units 6 and 7 responding to electrical stimulation of cutaneous receptive field. Upper trace shows single, and middle trace multiple, sweeps at $1-\mathrm{Hz}$ stimulus frequency. Lower trace shows one unit failing to respond to each stimulus delivered at $30 \mathrm{~Hz}$.

the dorsal horn. Three of these cells failed to follow high-frequency cutaneous stimulation and one proprioceptive cell had a long duration $(1.2 \mathrm{~ms})$ and biphasic $(800 \mu \mathrm{V})$ action potential waveform. Unit 3 was recorded from the edge of the dorsal horn; its exact position was uncertain.

Changes in Responsiveness during Behavior. On two separate occasions, constant-intensity electrical stimuli regularly evoked slow potentials and single cutaneous unit responses in the dorsal horn when the cat was not eating, but elicited no responses when the cat was eating. Both single units were postsynaptic and responded only to innocuous tactile stimuli. One example of this phenomenon and a histologic section including the unit's recording site are shown in Fig. 7. This cycle of changing responsiveness was reproducible and was not affected by the posture of the cat; it was not observed in any primary afferent fibers or in the 22 other tested postsynaptic cells.

\section{DISCUSSION}

Cell Classification. The results support the use of our principal criteria for the classification of somatosensory units into presynaptic (primary afferent) and postsynaptic elements in the intact spinal cord of the awake cat. The failure of a neural element to follow $100-\mathrm{Hz}$ peripheral stimulation at a con- 

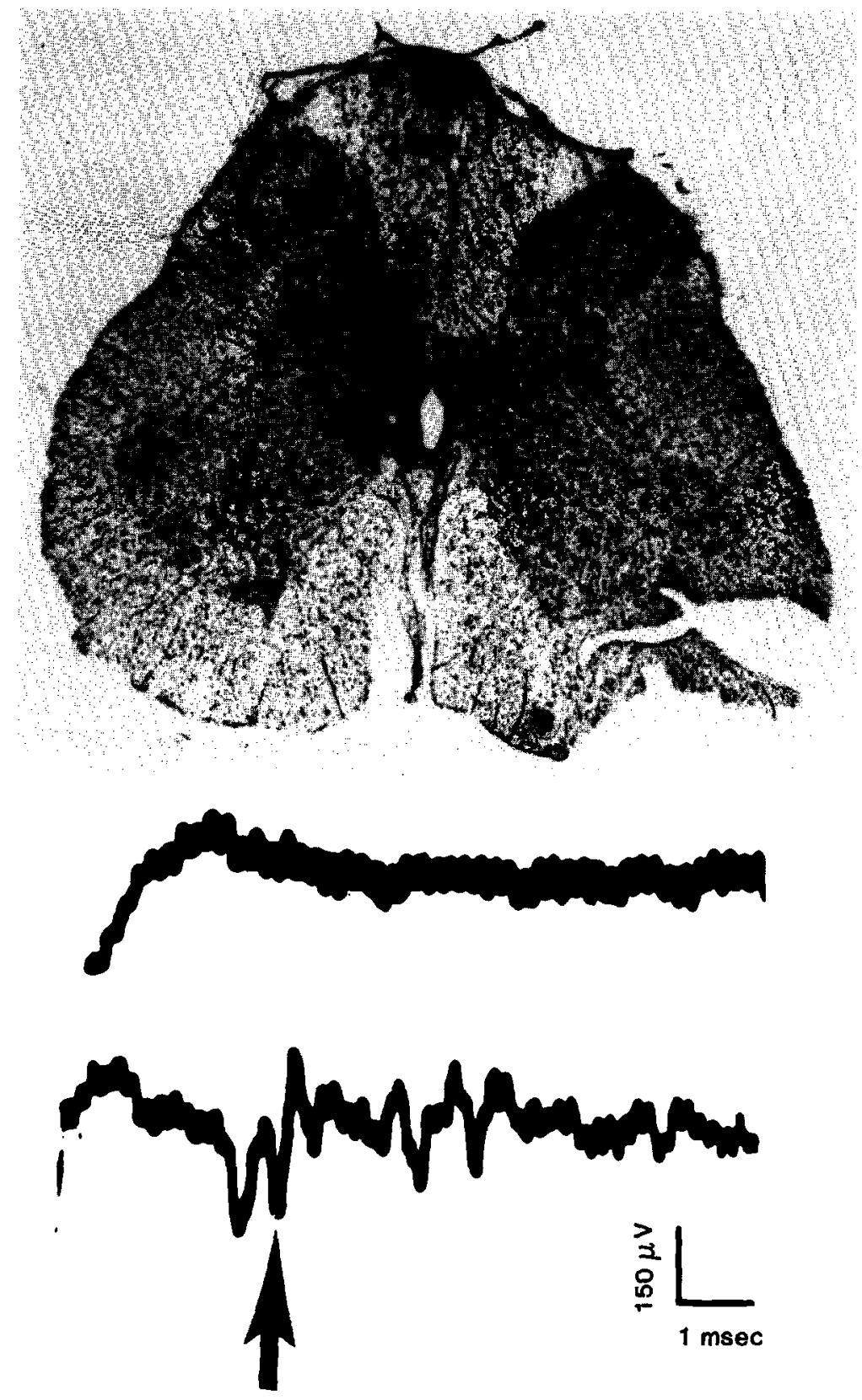

FIG. 7. Behavior-related modulation of unit responses to electrical stimulation (beginning of traces) of receptive field (distal tail). Histologic section shows loci of electrolytic lesions placed at site of unit recording (upper arrow) and end of track (lower arrow). Upper trace shows absence of unit responsc whilc cat was cating; lower trace shows sample of unit's (arrow) 80\% response ( 25 trials) when cat was not eating. 
stant latency has been considered sufficient to identify postsynaptic cells and distinguish most of them from presynaptic fibers $(10,34,39)$. Other principal criteria we used included an action potential waveform indicative of a cell body and multiple spikes evoked in response to a single, brief shock to the receptive field. The classification of units based on these three principal criteria was supported by the distribution of other physiologic properties. Thus, there were five secondary characteristics that were found either exclusively or primarily in the postsynaptic cell population: large receptive field size, conduction velocity of less than $20 \mathrm{~m} / \mathrm{s}$, recording site in gray matter, more than one type of adequate stimulus, and behaviorally associated changes in excitability. Based on our sample, it seems highly probable that any unit that has two or more of these five secondary characteristics is postsynaptic.

Spontaneous Activity. We observed no spontaneous activity in cutaneous primary afferent fibers. This is consistent with the low levels or lack of background discharge found in the cutaneous nerves of anesthetized animals (6) and humans $(25,35)$. Burgess et al. (8) also reported a lack of spontaneous activity in all but a subpopulation (5\%) of touch fibers. Resting discharge is seen only in muscle afferents (19), fibers from specific thermoreceptors (15), and afferent fibers from some slowly adapting mechanoreceptors $(20,21,23$, 25, 35).

In contrast to anesthetized and spinal animals, in which most second-order somatosensory cells are spontaneously active $(5,16,36)$, all isolated postsynaptic cells with cutaneous receptive fields that we recorded were silent in the absence of intentional stimulation. This was not an artifact produced by the experiment because these neurons were easily excited by peripheral stimuli. Furthermore, all the proprioceptive cells, including the seven classified as postsynaptic, were spontaneously active. Wall et al. (37), recording from lamina IV neurons of the awake and moving rat, also found an absence of spontaneous activity. Bromberg and Fetz (2) and Collins (11), recording in the awake monkey and cat, respectively, observed that most cutaneously activated spinal cord cells did not discharge spontaneously. The awake state thus resembles the decerebrate condition in which dorsal horn cells have less spontaneous activity than do those in the spinal animal, due to descending inhibition from the brain stem $(4,17)$. Anesthesia also inhibits at least some of the brain stem cell groups that project to the spinal cord (27) and may release the second-order projection neurons from inhibitory influences that are present in the awake animal.

Adequate Stimuli. The distribution of hair and touch primary afferent fibers found in this study is consistent with previous single-fiber recordings from cutaneous hind limb $(8,20)$ and tail nerves (29). Uddenberg (33) found similar proportions in the dorsal columns. Response characteristics were also like those found in cutaneous nerves (21); hair units were rapidly adapting, whereas all units that responded to skin displacement were slowly adapting. 
Only five (14\%) of the units classified as postsynaptic neurons showed evidence of convergent innervation from more than one type of receptor. This percentage of multireceptive cells is significantly lower than that reported for acute preparations $(5,38)$. It is possible that, due to a sampling bias, lamina IV (low-threshold) cells were encountered most frequently, but this is not supported by the histological reconstruction of our recording sites. The type of searching stimulus used may have contributed to the high percentage of cells which responded to only one type of low-threshold stimulus. Although each characterized cell was tested for a response to noxious pinch, it is possible that some of these cells would have been activated by noxious heat. Another possibility is that polymodal responsiveness is suppressed by descending inhibition in awake but not in anesthetized or surgically altered animals. Inhibition from the brain stem can change WDR cells into low-threshold units (36) and can change the adequate stimulus for hair and low-threshold cells (4). Soja and Sinclair (30) demonstrated that, in the intact, anesthetized cat, cold block of impulses descending in the dorsolateral funiculus selectively enhances nociceptive input to dorsal horn neurons. The relatively higher percentage of multireceptive postsynaptic cells found in the spinal cords of acute preparations, then, may be due to a release from this descending inhibition. Although recordings from the awake monkey (14) indicate that this may not be the case for neurons in the medullary dorsal horn, Collins and Rem (13) also found that multireceptive responses are reduced in the physiologically intact spinal cord. It is also possible that the periodic presentation of food in our experiments activated inhibitory influences descending from supraspinal sites.

Only one postsynaptic nociceptive-specific cell was found. This is not surprising because a low-intensity search stimulus was used. It implies nothing about the frequency of nociceptive-specific cells in the spinal cords of awake animals.

Receptive Field Size. One-third of the primary afferent fibers had receptive fields larger than $2 \mathrm{~cm}^{2}$ and several receptive fields on the tail were 6 to 8 $\mathrm{cm}^{2}$. Allowing for imprecision caused by working with an awake cat in a body cast, these receptive fields are still considerably larger than the 3 to 180 $\mathrm{mm}^{2}$ often described $(8,20,28)$. However, the majority of the receptive fields that we saw were located on the proximal limb, trunk, and tail. Receptive field size on both the forelimb and hind limb of the cat has been shown to increase with distance from the toes. Fields to $6 \mathrm{~cm}^{2}$ have been found on the upper portion of the hind limb (7) and a $64-\mathrm{cm}^{2}$ primary afferent receptive field was reported on the cat abdomen (3). In contrast, receptive field sizes on the tail are not organized with respect to distance from the trunk (29). Primary afferent receptive fields of several square centimeters are found throughout the length of the tail. 
Fiber Types. The estimated conduction velocities of the primary afferent fibers ranged from 23 to $100 \mathrm{~m} / \mathrm{s}$ with a mean of $53 \mathrm{~m} / \mathrm{s}$. This measurement places most of them in the A range. The four units with conduction velocities less than $30 \mathrm{~m} / \mathrm{s}$ could be fast A fibers. Unclassified units with a conduction velocity less than $20 \mathrm{~m} / \mathrm{s}$ are probably postsynaptic elements because extracellular metal microelectrodes are not likely to record from very fine afferent fibers in the central nervous system.

Behaviorally Related Modulation. The sensory responsiveness of primary afferent fibers was not correlated with the behavioral state of the animal. However, the activity of two (8\%) of the identified postsynaptic neurons was reduced when the cat's attention was directed toward the food, a condition known to significantly reduce behavioral responses to noxious thermal stimuli (9). Hayes et al. (14) observed behaviorally related changes in the response to noxious thermal stimuli by $66 \%$ of medullary dorsal horn nociceptive neurons in the monkey. The greater proportion of modulated cells recorded by Hayes et al. (14) could be due to both increased potential for modulation within pain pathways and the effect of behavioral training in the monkeys. Hyvarinen et al. (22) found that only $16 \%$ of primate S1 cortical neurons that responded to a vibratory stimulus were modulated when attentive behavior was directed toward the stimulus. These findings are more in accord with our results and suggest that behaviorally related modulation of neuronal excitability is readily detectable in a limited population of somatosensory neurons.

\section{REFERENCES}

1. Brenowitz, G. L., AND L. M. Pubols. 1981. Increased receptive field size of dorsal horn neurons following chronic spinal cord hemisections in cats. Brain Res. 216: 45-59.

2. BRomberG, M. B., AND E. E. FETZ. 1977. Responses of single units in cervical spinal cord of alert monkeys. Exp. Neurol. 55: 469-482.

3. Brown, A. G. 1968. Cutaneous afferent fibre collaterals in the dorsal columns of the cat. Exp. Brain Res. 5: 293-305.

4. BRown, A. G. 1971. Effects of descending impulses on transmission through the spinocervical tract. J. Physiol. (London) 219: 103-125.

5. BRown, A. G., AND D. N. FRANZ. 1969. Responses of spinocervical tract neurones to natural stimulation of identified cutaneous receptors. Exp. Brain Res. 7: 213-249.

6. BROWN, A. G., AND A. IGGO. 1967. A quantitative study of cutaneous receptors and afferent fibres in the cat and rabbit. J. Physiol. (London) 193: 707-733.

7. Brown, P. B., AND H. R. KoERBER. 1978. Cat hindlimb tactile dermatomes determined with single-unit recordings. J. Neurophysiol. 41: 260-267.

8. Burgess, P. R., D. Petit, AND R. M. WARREN. 1968. Receptor types in cat hairy skin supplied by myelinated fibers. $J$. Neurophysiol. 31: 833-848.

9. CASEY, K. L., AND T. J. MORROW. 1983. Nocifensive responses to cutaneous thermal stimuli in the cat: stimulus-response profiles, latencies, and afferent activity. $J$. Neurophysiol. 50: $1497-1515$.

10. Christensen, B. N., AND E. R. PerL. 1970. Spinal neurons specifically excited by noxious or thermal stimuli: marginal zone of the dorsal horn. $J$. Neurophysiol. 33: 293-307. 
11. Collins, J. G. 1983. Neuronal activity recorded from the spinal cord dorsal horn of physiologically intact, awake, drug-free, restrained cats: a preliminary report. Brain Res. 322: 301-304.

12. ColluNs, J. G. 1985. A technique for chronic extracellular recording of neuronal activity in the dorsal horn of the lumbar spinal cord in drug-free, physiologically intact cats. $J$. Neurosci. Methods 12: 277-287.

13. COLLINS, J. G., AND K. E. REM. 1986. The probability of spinal dorsal horn neurons having WDR response profiles is low in the physiologically intact cat. Soc. Neurosci. Abstr. 12: 224.

14. Hayes, R. L., R. Dubner, AND D. S. Hoffman. 1981. Neuronal activity in medullary dorsal horn of awake monkeys trained in a thermal discrimination task. II. Behavioral modulation of responses to thermal and mechanical stimuli. J. Neurophysiol. 46: 428443.

15. HENSEL, H., A. IGGO, AND I. WITT. 1960. A quantitative study of sensitive cutaneous thermoreceptors with C afferent fibres. J. Neurophysiol. 153: 113-126.

16. Hillman, P., AND P. D. WALl. 1969. Inhibitory and excitatory factors influencing the receptive fields of lamina 5 spinal cord cells. Exp. Brain Res. 9: 284-306.

17. HOLMQVIST, B., AND A. LUNDBERG. 1959. On the organization of the supraspinal inhibitory control of interneurones of various spinal reflex arcs. Arch. Ital. Biol. 97: 340-356.

18. HubeL, D. H. 1960. Single unit activity in lateral geniculate body and optic tract of unrestrained cats. J. Physiol. (London) 150: 91-104.

19. HUNT, C. C. 1954. Relation of function to diameter in afferent fibres of muscle nerves. $J$. Gen. Physiol. 38: 117-131.

20. Hunt, C. C., ANd A. K. McINTyre. 1960. Properties of cutaneous touch receptors in cat. J. Physiol. (London) 153: 88-98.

21. HunT, C. C., AND A. K. MCINTYRE. 1960. An analysis of fibre diameter and receptor characteristics of myelinated afferent fibres in cat. J. Physiol. (London) 153: 99-112.

22. HyVARINEN, J., A. PORANEN, AND Y. JOKINEN. 1980. Influence of attentive behavior on neuronal responses to vibration in primary sensory cortex of the monkey. J. Neurophysiol. 43: 870-882.

23. IGGO, A., AND A. R. MUIR. 1969. Structurc and function of a slowly adapting touch corpuscle in hairy skin. J. Physiol. (London) 200: 763-796.

24. Kitahata, L. M., I. Sato, ANd A. Taub. 1971. Lamina-specific suppressions of dorsal horn unit activity by nitrous oxide and by hyperventilation. J. Pharmacol. Exp. Ther. 176: $101-108$.

25. KNIBESTOL, M., AND A. B. VALLBO. 1970. Single unit analysis of mechanoreceptor activity from the human glabrous skin. Acta Physiol. Scand. 80: 178-195.

26. Morkow, T. J. 1980. Improved technique for recording single unit activity in awake animals. Brain Res. Bull. 5: 91-93.

27. MORROW, T. J., AND K. L. CASEY. 1983. Suppression of bulboreticular units to analgesic mesencephalic stimulation. Somatosensory Res. 2: 151-168.

28. Petit, D., AND P. R. Burgess. 1968. Dorsal column projection of receptors in cat hairy skin supplied by myelinated fibers. J. Neurophysiol. 31: 849-855.

29. REID, K. H. 1970. Dermatomes and skin innervation density in the cat's tail. Exp. Neurol. 26: $1-16$.

30. SOJA, P. J., AND J. G. SINCLAIR. 1983. Tonic descending influences on cat spinal cord dorsal horn neurons. Somatosens. Res. 1: 83-93.

31. SORKIN, L. S., T. J. MORROW, AND K. L. CASEY. 1981. Spinal cord unit activity: behaviorally related changes in awake cat. Pain 1(Suppl.): S128.

32. SORKIN, L. S., T. J. MORROW, AND K. L. CASEY. 1983. Spinal cord unit activity in awake cat. Soc. Neurosci. Abstr. 9: 473. 
33. UDDENBERG, N. 1968. Differential localization in dorsal funiculus of fibres originating from different receptors. Exp. Brain Res. 4: 367-376.

34. UDDENBERG, N. 1968. Functional organization of long, second order afferents in the dorsal funiculus. Exp. Brain Res. 4: 377-382.

35. Vallbo, A. B., AND K.-E. HaGbarth. 1968. Activity from skin mechanoreceptors recorded percutaneously in awake human subjects. Exp. Neurol. 21: 270-289.

36. WALL, P. D. 1967. The laminar organization of dorsal horn and effects of descending impulses. J. Physiol. (London) 188: 403-423.

37. WALL, P. D., J. FREEMAN, AND D. MAJOR. 1967. Dorsal horn cells in spinal and in freely moving rats. Exp. Neurul. 19: 519-529.

38. WALL, P. D., E. G. MerRILL, AND T. L. YAKSH. 1979. Responses of single units of laminae 2 and 3 of cat spinal cord. Brain Res. 160: 245-260.

39. WINTER, D. L. 1965. N. gracilis of cat. Functional organization and corticofugal effects. $J$. Neurophysiol. 28: 48-70. 